\title{
LES SOIES DOUBLÉES CHEZ LES LARVES D'ANOPHELES
}

\author{
Par J. GAUD et J. LAURENT
}

En mars 1950, l'un de nous capturait à Beni-Mellal (Atlas central marocain) une larve d'A. claviger Meigen présentant une anomalie assez rare. Cette larve présente quatre soies clypéales antéro-internes, égales en longueur et en diamètre, implantées sur des tubercules basilaires normaux, distincts équidistants (voir pl. ci-contre).

Cette trouvaille nous incite à reprendre la question de la duplication des soies clypéales chez les larves d'anophèles. En 1947, l'un de nous (1) avait décrit quatre larves présentant cette anomalie et rapporté celle-ci, non sans réserve, à une ébauche de monstruosité double symétrique. Depuis lors, nous avons observé assez souvent des anomalies de cet ordre. Les 28 cas que nous avons essayés de classer dans le tableau ci-après correspondent à l'examen de 26.000 larves.

Si la fréquence globale de l'anomalie est de l'ordre de 1 p. 1.000 , il semble exister, à ce point de vue, de fortes différences selon les espèces. Nous avons déjà noté, en 1947, que la duplication des soies clypéales semblait s'observer surtout chez les larves du sous-genre Anopheles stricto sensu. Ceci paraît se confirmer.

Nous relevons :

- 23 anomalies sur 15.000 larves du S.G. Anopheles,

- 5 anomalies sur 11.000 larves du groupe Myzomyia.

L'espèce $A$. claviger semble particulièrement prédisposée : 13 anomalies pour un peu plus de 5.000 larves examinées, soit 2,5 p. 1.000 .

La duplication s'observe sur les trois groupes de soies clypéales. Toutefois, elle est plus fréquente sur les soies antéro-internes (13 cas) qu'elle ne l'est sur les soies antéro-externes (8 cas) et surtout sur les soies postérieures ( 2 cas).

Le siège de l'anomalie est plus souvent à droite (13 cas) qu'à gauche (7 cas).

(1) J. GAud. - Ann. de Paras., XXII, 1947, 394.

Annales de Parasitologie, T, XXV, Nos 5-6, 1950 , 
T. XXV, No 5-6, 1950
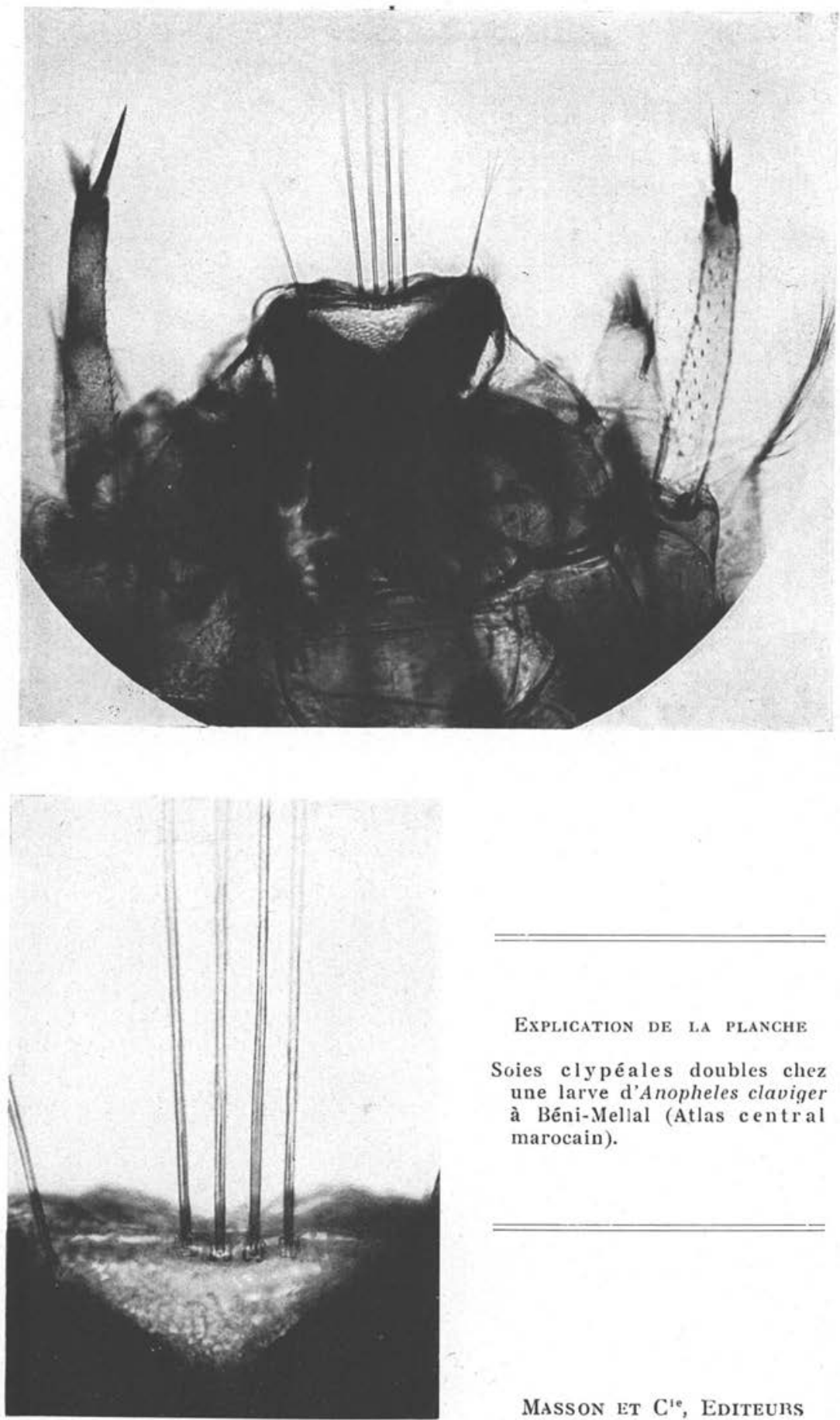

Explication DE IA PLANCHE

Soies clypéales doubles chez une larve d'Anopheles claviger à Béni-Mellal (Atlas central marocain).

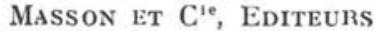




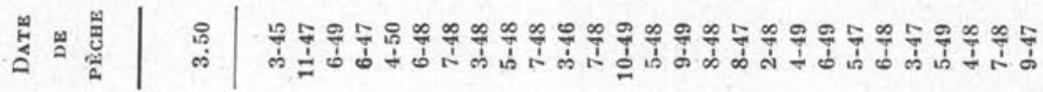

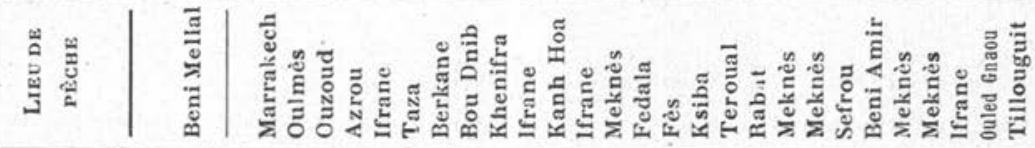

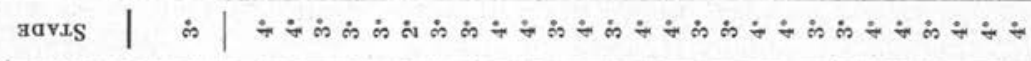

落|

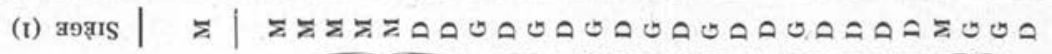

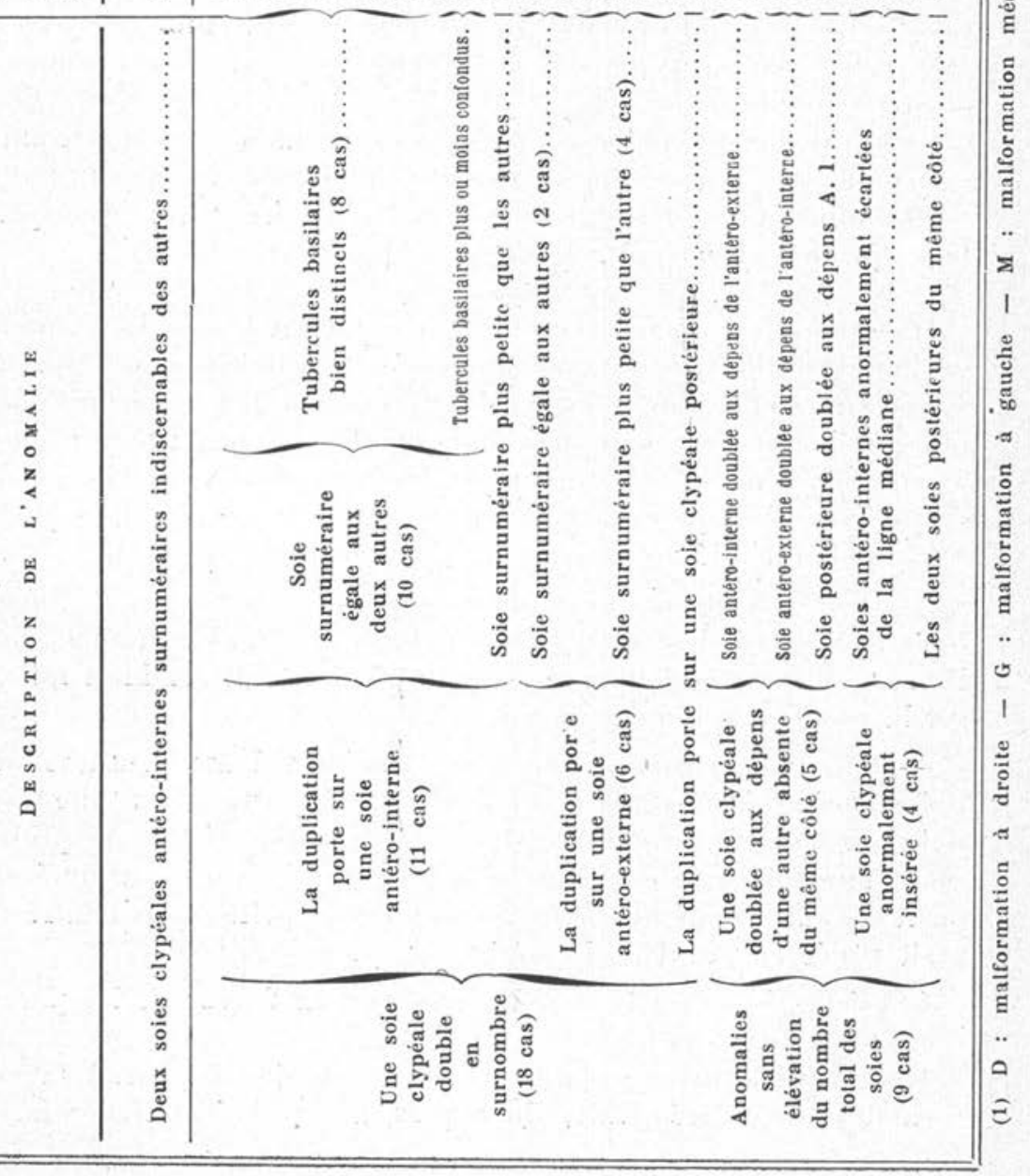


Les larves anormales paraissent provenir indifféremment de toutes les régions du Maroc et être pêehées en n'importe quelle saison.

Interprétation de l'anomalie. - Nous avions d'abord été tentés d'interpréter ces anomalies comme une monstruosité double isolée. Nous pensons maintenant qu'il s'agit d'une malformation de la soie et non d'une malformation de l'embryon.

L'hypothèse monstruosité double rend compte de façon satisfaisante de la prédominance de l'anomalie sur les soies clypéales antéro-internes. C'est aussi une explication plausible de la fréquence plus grande de l'anomalie chez les Anopheles S.S. Une séparation plus accusée des extrémités céphaliques jumelles serait nécessaire pour faire apparaître le phénomène chez les Myzomyia. Cette séparation plus accusée serait peut-être incompatible avec la survie de la larve jusqu'au $3^{\circ}$ ou $4^{\circ}$ stade, ceux que l'on examine le plus généralement.

En revanche, l'hypothèse monstruosité double rend mal compte des anomalies classées dans la moitié inférieure du tableau; rend mal compte aussi des différences de fréquence entre les espèces maculipennis et claviger.

Dans notre première relation, nous avions noté que la fréquence des duplications portant sur les soies clypéales pouvait être une simple apparence, ces soies, d'intérêt taxonomique important, étant plus fréquemment examinées que d'autres soies. Depuis, nous avons recherché systématiquement toute duplication de l'ensemble des soies céphaliques et thoraciques sur 510 larves d'anophèles. Nous avons décelé :

- Deux soies frontales doublées, l'une chez une larve d'A. maculipennis au $4^{\circ}$ stade, l'autre chez une larve d'A. turkhudi au $4^{\circ}$ stade. Dans les deux cas, l'anomalie siégeait à droite et semblait intéresser la soie frontale moyenne (fig. 2).

- Deux soies du palpe maxillaire doublées, l'une chez une larve d'Anopheles claviger au $4^{\circ}$ stade (fig. 1), l'autre chez une larve d'Anopheles turkhudi au $4^{\circ}$ stade. L'anomalie siégeait respectivement une fois à gauche, une fois à droite. La soie surnuméraire, égale à l'autre dans un cas, légèrement plus petite dans l'autre cas, était située en avant de la soie normale.

Ces deux dernières trouvailles sont peu en faveur de l'hypothèse d'une monstruosité double.

Enfin, l'hypothèse de la monstruosité double supposait la malformation aussi fréquente, sinon plus, aux premiers stades larvai- 


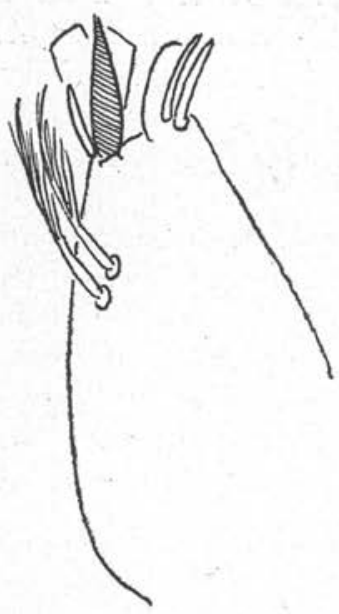

FIg. 1. - Paipe maxillaire gauche’ avec soie externe doublée chez une larve d'Anopheles claviger.

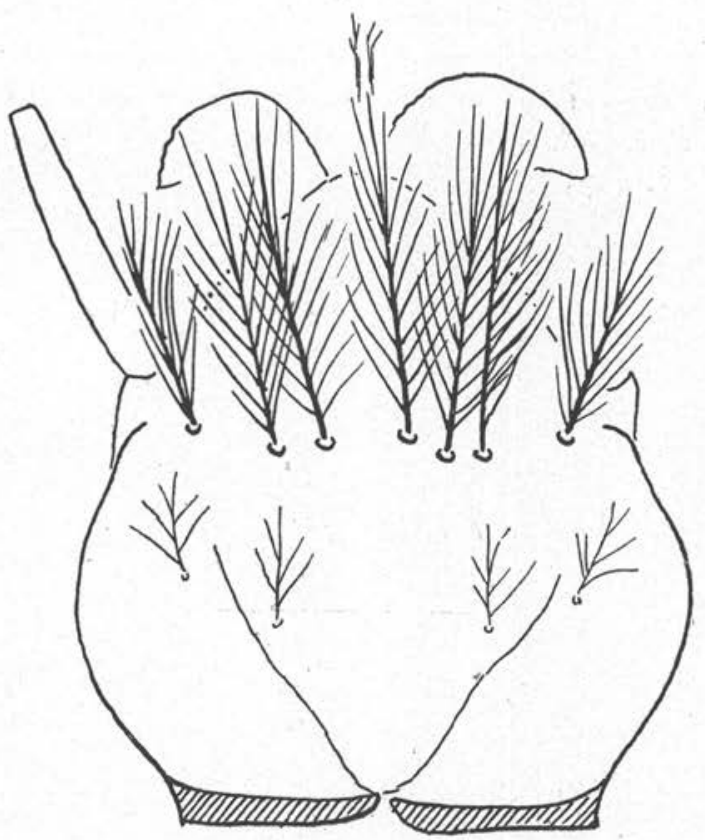

FIG. 2. - Tête d'une larve d'Anopheles maculipennis avec la soie frontale moyenne droite doublée 
res qu'aux troisième et quatrième. Or, nous avons cherché vainement une duplication sur 5.000 larves (A. maculipennis et A. claviger) aux premier et second stades. La valeur de cette observation négative est cependant diminuée du fait que ces larves représeniaient la descendance d'un nombre relativement restreint de femelles ayant pondu en captivité.

Remarquant que les soies intéressées par des duplications sont généralement des soies antérieures et proéminentes, et suspectant le rôle d'un traumatisme du bourgeon dans la genèse de la malformation, nous avons essayé de pratiquer l'ablation de quelques soies frontales sur des larves au $2^{\circ}$ stade, dans l'espoir de voir apparaître, après la mue, des duplications anormalement fréquentes. Mais le petit nombre de larves ayant survécu au traumatisme ne nous a pas permis une vérification de l'idée.

\section{RÉSUMÉ}

Description et étude statistique de 31 anomalies des soies céphajiques chez des larves d'anophèles, dont 28 duplications. Exposé d'éléments d'interprétation de ces anomalies dans le sens d'une malformation du bourgeon de la soie et non d'une malformation générale de l'embryon.

Institut d'Hygiène du Moroc, Rabat, Maroc. 